\title{
Moving FITS Training from the Laboratory to the Flight Line
}

\author{
Wendy S. Beckman, Mark N. Callender, Steve Gossett, \\ Wayne A. Dornan and Paul A. Craig
}

\begin{abstract}
In 2004, Middle Tennessee State University (MTSU) first conducted training on an experimental basis using a FAA-Industry Training Standards (FITS) accepted Private/Instrument curriculum. Based on the results of that and subsequent studies, the decision was made to move to full-scale implementation of this Private/Instrument curriculum beginning in January of 2007. Before this course of action was chosen, a number of issues were resolved including flight school preparation, development of a Commercial FITS approved syllabus, aircraft scheduling and training time considerations, and changes to the original syllabus. Since implementation as the standard MTSU flight school curriculum, sixteen students have completed the course. The mean flight times and number of setbacks experienced by students during training continue to compare favorably to traditional training methods.
\end{abstract}

\section{INTRODUCTION}

In 1998, the FAA announced a "SAFER SKIES” initiative to achieve significant reductions in the number of General Aviation (GA) fatal accidents by 2007 (United States General Accounting Office, 2000). As part of this initiative, the General Aviation Joint Steering Committee (GAJSC) focused on the leading causes of GA accidents. In order to assess what new safety challenges occur with the advent of the Technically Advanced Aircraft (TAA), the GAJSC established a TAA study team to investigate safety issues with TAA aircraft (Dornan, Beckman, Gossett, \& Craig, 2007b; Fiduccia et al, 2003). Part of the impetus for this was an observed increase in fatal accidents with the next generation TAA's in the early to mid-90's (AOPA Air Safety Foundation, 2005; National Transportation Safety Board (NTSB), 1990). A major recommendation in this report was that the current training format in the industry was insufficient to exploit the additional safety features of TAA, and that there was a critical need to develop a TAA training program in the GA community (Fiduccia et al, 2003). As a result of these recommendations, the FAA implemented the FAA-Industry Training Standards (FITS) program (Federal Aviation Administration, 2004; Glista, 2003). This program emphasizes the importance of "real world" training exercises in the form of scenario training. This approach had proven successful in the air carrier industry, but had not been attempted in the GA community. This training places a major emphasis on: aeronautical decision making skills, risk management, situational awareness, and single pilot resource management using real-time flight scenarios (Ayers, 2006; Glista, 2003). Studies from Embry-Riddle Aeronautical University (ERAU), the University of North Dakota (UND), and Middle Tennessee State University (MTSU) on the effectiveness of the FITS curriculum have resulted in the FAA accepting the FITS training approach as the industry standard for all future flight training in General Aviation (Glista, 2003).

In 2004, MTSU received the first FAA acceptance to train students for a combined Private Certificate/Instrument rating in TAA using the FITS training program. This was a novel approach, as traditionally, a student pilot is required to first complete training for a Private Certificate, then complete additional training for an Instrument rating. This FITS curriculum was developed by ERAU and UND through the FAA Air Transportation Center of Excellence for General Aviation (CGAR). The FITS curriculum was first tested at MTSU in 2004-05 in a NASA funded project called "SAFER." Over the last three years, the MTSU SAFER research team has published and presented the results of several studies that have indicated the effectiveness of the FITS approach for not just TAA, but for use in all aircraft (Craig, Bertrand, Dornan, Gossett, \& Thorsby, 2005a, 2005b; Dornan, Beckman, Gossett, \& Craig, 2007a; Dornan, Beckman, Gossett et al., 2007b; 
Dornan, Beckman, Gossett, Craig, \& Mosey, 2007; Dornan, Craig, Beckman, \& Gossett, 2007; Dornan, Craig, Gossett, \& Beckman, 2006; Dornan, Craig, Gossett, Beckman, \& Mosey, 2007; Dornan, Gossett, Craig, \& Beckman, 2006). Each of these studies was conducted in a somewhat controlled environment, in that small sub-sets of the entire population of Professional Pilot students at MTSU were utilized. Given the success of these experimental groups, the decision was made to move forward with full scale implementation of the FITS Private/Instrument curriculum for all students at the MTSU flight school. For several decades, the MTSU flight program had used a traditional training approach in which students obtained the Private Pilot Certificate first, followed by a semester gaining VFR cross country experience, followed by a semester earning the Instrument Rating. In January 2007, the MTSU Aerospace Department changed this conventional, maneuver-based methodology to a combined Private and Instrument syllabus that has received FITS acceptance. The decision to implement the FITS curriculum for all students in the program required a great deal of decision making, implementation planning, and flight instructor training (Dornan, Beckman, Gossett, Craig et al., 2007). There were five significant issues that were addressed before the implementation decision was made: Flight school preparation, the availability of a FITS Commercial Pilot syllabus, the use of both TAA and conventional aircraft, the length of time required for course completion, and the changes to be made to the original syllabus used in the NASA SAFER project.

\section{Flight School Preparation}

It had been hoped that the MTSU flight program would be ready to implement the FITS Private/Instrument syllabus as the standard training methodology in August of 2006, but ultimately the changeover was delayed until January 2007. This delay allowed the flight school staff the necessary time to address the issues that the FITS syllabus would create. These issues have been described in a previous publication (Dornan, Beckman, Gossett, Craig et al., 2007), but were in large part flight instructor training, development of ground school curriculum, and aircraft scheduling.

\section{Development of a FITS Commercial Syllabus}

The students who participated in the experimental FITS groups were all enrolled in the MTSU Professional Pilot program, which requires students to obtain the FAA Commercial Pilot Certificate and Multiengine Rating prior to graduation. Therefore, the students who completed the FITS Private/Instrument syllabus then utilized a traditional Commercial Pilot course to complete the Commercial Certificate and to meet graduation requirements. It was frustrating for students who had learned with scenario-based training to have to revert to maneuver-based training, especially when a number of the Commercial maneuvers (chandelles, lazy 8's) seemed to have little relevance to actual commercial operations. In addition, any reductions achieved in flight hours required in Private/Instrument training were quickly lost when the students went back to a syllabus requiring minimum flight times. Thus, it was decided that the Private/Instrument syllabus would not be used as the standard curriculum until there was available a FITS Commercial syllabus as a complement. This would allow the students to complete their entire training requirement using the FITS methodology, therefore maintaining all the benefits produce by FITS. In early 2007, MTSU was tasked by CGAR to write a FITS Commercial Syllabus. In the summer of 2007 the syllabus was reviewed by a national review team and ultimately awarded FITS acceptance (Federal Aviation Administration, 2007a, 2007b). It was then possible to link the FITS Private/Instrument syllabus with the FITS Commercial syllabus so that students could go from their very first flight lesson through Commercial Pilot using the FITS methodology.

\section{Use of Both TAA and Conventional Aircraft}

The students in the SAFER project trained exclusively in TAA. Since MTSU's training fleet is only 20\% TAA, to use the FITS syllabus for the entire program it became necessary to use the FITS syllabus with both TAA and conventional aircraft. Some lessons contained within the syllabus were designated as "TAA lessons" which required use of a TAA. Most 
lessons however, did not designate TAA and those lessons could be completed in either a TAA or conventional aircraft. The decision to use both TAA and non-TAA interchangeably was a matter of necessity, not a research question. The flight school needed to utilize $100 \%$ of the fleet to accommodate the schedule, so to implement the FITS syllabus for all students; every airplane had to be used.

\section{Length of Training Time}

The students in the original SAFER project found it difficult to complete the Private/Instrument syllabus in a single semester. While there were several students who did complete in one semester, on average more time was needed. Therefore the decision was made to allow students two semesters to complete the FITS Private/Instrument syllabus. This produces some challenges when students begin the curriculum in the spring semester, and then are not able to stay for the summer semester. For those unable to remain in school during the summer, an undesirable gap in the middle of training occurs. There is no easy solution to this problem, beyond encouraging students entering the program to either begin training in the fall semester or to plan on staying for the summer semester, so that their training will be continuous.

\section{Improvements to the Original Syllabus}

MTSU was the first to use the syllabus produced by CGAR, and as a result became the beneficiary of a number of "lessons learned." Before the decision was made to use the FITS syllabus as the standard training curriculum several changes were made to the syllabus that reflected these lessons learned. It was found that there was not enough landing practice experienced by students by performing only a landing at a scenario destination and a landing when returning to the home airport. Provisions were made in the syllabi for lessons to contain multiple landings at both the destination and home airport on several lessons. It was also discovered that students who only experienced an instrument approach at the end of a relatively long cruise portion of flight, with abundant time for approach set up, had difficulty when expected to prepare for an approach on short notice. Thus, guidance was given to instructors to include scenarios where the expected approach was changed at the last minute, due to ATC needs or weather changes, in order to increase student proficiency in dealing with such changes. Greater emphasis was also placed on conducting GPS approaches, while references to NDB approaches were removed, as the aircraft in use were not ADF-equipped.

While the number of lessons remained the same as in the original, on some lessons the flexibility to conduct the training in either a Flight Training Device (FTD), a DA-20, a conventional DA-40, or a G-1000 equipped DA40 was added. This allowed instructors the flexibility to move between equipment types as availability allowed, and enhanced students learning by exposing them to a variety of equipment. In addition, instead of providing a detailed listing of all tasks to be completed on a flight, the task listing was shortened to those tasks being stressed in that particular lesson. For instance, items such as engine start up, taxiing, and the pre-takeoff check are key tasks in the early lessons, but by a point mid-way through the curriculum, those tasks should have already been mastered. While these tasks are still important, other skills are the focus of later lessons. This revision was necessary as the sheer amount of text on each lesson page was overwhelming to flight instructors, and they had difficulty determining the intent of a particular lesson.

\section{RESULTS}

There were sixteen students who started the FITS syllabi in January of 2007, and who had completed the FITS syllabi by October 2007. As indicated in the Introduction, the curriculum was designed to take two semesters, so only students who were willing to stay at school and fly during the summer were expected to be completed. There were two students who came very close to completing the syllabus in one semester, as they only required a few days after the spring semester to complete their training. The remainder of the students completed during the summer months.

To determine the effectiveness of the fullscale implementation, the average flight times and setbacks of these 16 students was compared to both the original SAFER project experimental 
group and to a group of conventional syllabus students which were used for comparison purposes in the first MTSU FITS study (Craig et al., 2005a). There were fifteen students in the original SAFER group, and sixteen students in the conventional syllabus group. As can be seen in Figure 1, the mean flight time for the fullscale implementation group to obtain the Private/Instrument was 95 hours. This compares to a mean of 88 hours for the SAFER experimental group, and a mean of 135 hours for the conventional syllabus students.

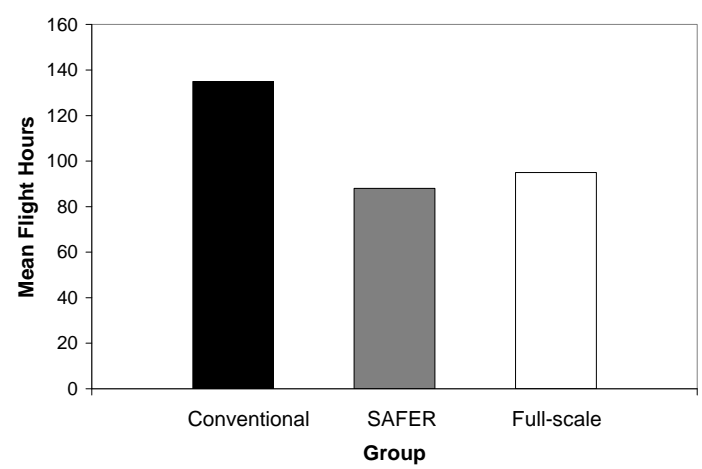

Figure 1: Mean hours to complete the Private Certificate with Instrument Rating for each of the three groups

A setback was defined as a lesson that a student had to repeat to obtain proficiency. As can be seen in Figure 2, the mean number of setbacks experienced by each group prior to obtaining the Private/Instrument were examined. For the full-scale implementation group, a mean of 12 setbacks was found. For the SAFER experimental group a mean of 6.91 setbacks was found, while the conventional syllabus group experienced a mean of 22.45 setbacks.

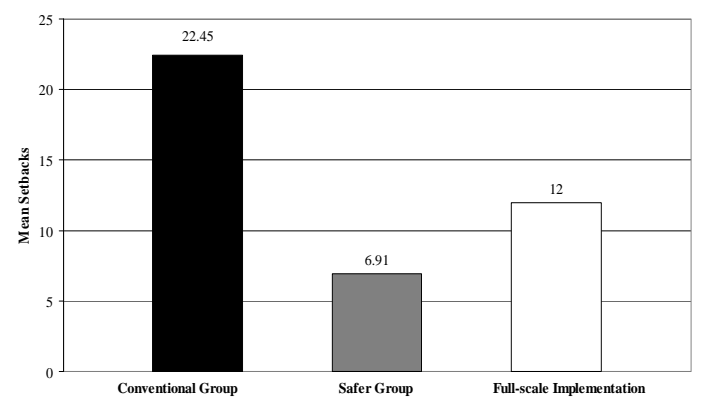

Figure 2: Mean number of setbacks experienced during training for each of the three groups
The setbacks were further examined to determine if there was a significant difference between pre-solo training setbacks and post-solo training setbacks. As can be seen in Figure 3, a 2X3 ANOVA revealed a significant interaction of training group versus pre-solo and post-solo setbacks $(\mathrm{F}=10.38, \mathrm{p}<0.01)$.

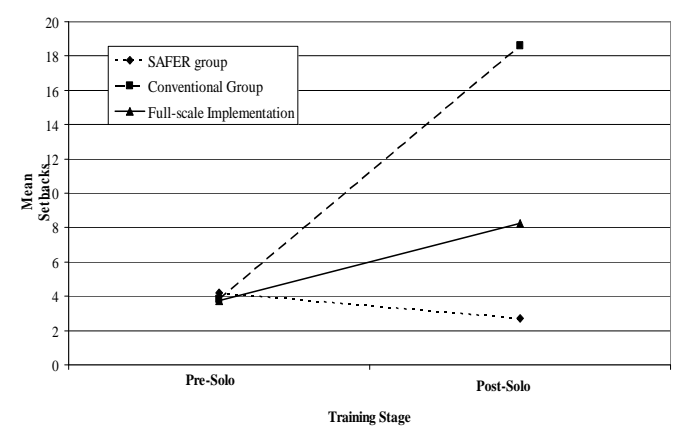

Figure 3: Mean number of setbacks for each group both pre-solo and post-solo

As can been seen from this figure the average number of pre-solo setbacks were not statistically different $(X=3.85$ for the conventional syllabus group, $X=4.21$ for the SAFER group, and $\mathrm{X}=3.75$ for the full scale implementation group). However, post-solo setbacks increased significantly in the conventional syllabus group $(X=18.6)$ and in the full implementation group ( $\mathrm{X}=8.25)$, versus the SAFER group ( $\mathrm{X}=2.71)$. A post hoc analysis revealed an overall significant difference in the conventional syllabus group compared to both FITS groups. Interestingly, with a post-solo setback mean of 8.25 , the full implementation group had a mean midway between the conventional syllabus group and the SAFER experimental group. Lastly, post hoc analysis revealed that the SAFER experimental group had significantly fewer post-solo setbacks compared to the other groups.

\section{DISCUSSION}

Based on the results above, it is evident that the students who have completed training thus far as part of the full-scale MTSU flight school implementation have experienced fewer setbacks and attained their private pilot certificate and instrument rating in fewer hours than the traditionally trained students that were used for comparison purposes. This outcome agrees with the results of the highly controlled 
SAFER project, which implemented FITS in a more research oriented environment. If the results of the SAFER project are used for comparison as the "ideal" situation, the full implementation comes very close to being ideal, although the mean number of setbacks and flight hours were slightly higher. This did not come as a surprise, in light of the challenges associated with implementing any program on a larger scale. Although it has been shown that the FITS syllabus and not simply training in a TAA is the source of improvements among students (Dornan et. al., 2006), SAFER students did experience the benefit of training solely in TAAs. Full implementation necessitated the use of both two conventional (DA-20 and DA-40) and TAA (DA-40 with G-1000) aircraft for training. The lack of a common aircraft from flight to flight is a confounding and likely detrimental variable.

Also, the SAFER project employed a small group of experienced and FITS trained instructors. The full implementation required the services of all flight school instructors who, although qualified, did not have a high experience level upon which to draw for scenario-based training. Along with low experience levels, they also were largely traditionally trained and so had not experienced the benefits of FITS training themselves. It is believed that as FITS trained pilots enter the instructor ranks in the near future, they will be both more prepared for and more comfortable with scenario-based training. It must be recognized that the full implementation did reduce the average number of pre-solo setbacks when compared to the SAFER project, although not by a statistically significant amount. The average pre-solo setbacks in the SAFER project was $X=4.21$, while the average pre-solo setbacks in the full implementation group was $X=3.75$. This improvement is believed to be the result of increasing the number of landings conducted in the pre-solo lessons, thereby providing more practice of the maneuver-based landing skills. The analysis of the mean setbacks experienced both pre-solo and post-solo was undertaken to assist in scrutinizing the syllabus for possible future revisions. It was found that many students in the full implementation group experienced setbacks just prior to the IFR stage check
(Lesson 19), when all of the instrument skills previously learned were being consolidated. This could indicate the need for an additional lesson prior to this point in the curriculum.

\section{CONCLUSION}

In closing, the first full implementation of the FITS private/instrument combined training course provided significant improvements with regards to both mean flight time and mean setbacks experienced versus traditional training methods. When coupled with rising training costs, this translates to savings for students. It should be noted that at the time of this writing, the sample size of students who have completed the curriculum is very small, which is a significant limitation of the study. However, this project is continuing, and data will continue to be collected as more students enroll in and complete the Private/ Instrument FITS syllabus. Data collection will also begin as students enter the Commercial FITS curriculum, to assist in determination of the effectiveness of that training course. This data will be made available in future articles.

Finally, reductions in setbacks, hours, and costs should not overshadow the original purpose for scenario-based/FITS training. The development of these curricula was motivated and driven by the desire to make flight training more effective in terms of producing a pilot who is better able to make safe decisions. Previous studies have shown that FITS trained pilots "make better decisions", are "more comfortable in the IFR environment", and are "more cautious" than traditionally trained pilots (Dornan et. al., 2006). It is primarily for these reasons that the FITS methodology should be considered, while the reduction in training costs are a secondary benefit. 


\section{REFERENCES}

AOPA Air Safety Foundation. (2005). Technically advanced aircraft: Safety and training. Frederick, MD: AOPA Air Safety Foundation.

Ayers, F. H. (2006). The application of scenario based recurrent training to teach single pilot resource management (SRM) under the FAA Industry Training Standards (FITS) Program. Journal of Aviation and Aerospace Research, 3, 13-25.

Craig, P. A., Bertrand, J. E., Dornan, W., Gossett, S., \& Thorsby, K. K. (2005a). Ab Initio training in the glass cockpit era: New technology meets new pilots. Paper presented at the International Symposium on Aviation Psychology.

Craig, P. A., Bertrand, J. E., Dornan, W., Gossett, S., \& Thorsby, K. K. (2005b). Scenario-based Private/Instrument syllabus versus traditional maneuver-based syllabi: A preliminary descriptive analysis. Collegiate Aviation Review, 23, 23-27.

Dornan, W. A., Beckman, W., Gossett, S., \& Craig, P. A. (2007). A FITS scenario-based training program enhances GPS pilot proficiency in the general aviation pilot. Collegiate Aviation Review, 25(1), 19-27.

Dornan, W. A., Beckman, W., Gossett, S., Craig, P. A., \& Mosey, P. (2007). The Implementation Of The FAA Industry Training Program In Technically Advanced Aircraft (TAA): Lessons Learned. Paper presented at the International Symposium on Aviation Psychology, Dayton, Ohio.

Dornan, W. A., Craig, P. A., Beckman, W., \& Gossett, S. (2007). Incorporating a Novel Approach using the Technically Advanced Aircraft. Paper presented at the 78th Annual Aerospace Medical Association Meeting.

Dornan, W. A., Craig, P. A., Gossett, S., \& Beckman, W. (2006). Best evidence for the FAA Industry Training Standards program for pilot training in technically advanced aircraft. Collegiate Aviation Review, 24(1), 58-66.

Dornan, W. A., Craig, P. A., Gossett, S., Beckman, W., \& Mosey, P. (2007). FAA Industry Training Standards (FITS) Program for Pilot Training: An MTSU Progress Report. Paper presented at the National Transportation Aviation Symposium.

Dornan, W. A., Gossett, S., Craig, P. A., \& Beckman, W. (2006). Best evidence on FITS. Paper presented at the National Training Aircraft Symposium, Daytona Beach, Florida.

Federal Aviation Administration. (2004). FAA-Industry Training Standards (FITS) Program 2004. Retrieved, from the World Wide Web: http://www.faa.gov/education_research/training/fits

Federal Aviation Administration. (2007a, September). FITS Generic Commercial Airplane Multi Engine Land Syllabus. Retrieved, from the World Wide Web: http://www.faa.gov/education_research/ training/fits/training/generic/media/commercial_amel.pdf

Federal Aviation Administration. (2007b, July). FITS Generic Commercial Airplane Single Engine Land Syllabus. Retrieved, from the World Wide Web: http://www.faa.gov/education research/ training/fits/training/generic/media/commercial_asel.pdfFiduccia et al. (2003). General Aviation Technically Advanced Aircraft FAA-Industry Safety Study, Final Report. Retrieved April 10, 2006, from the World Wide Web: http://www.faa.gov/ education_research/training/fits/research

Glista, T. (2003, March/April). FAA/Industry Training Standards (FITS): Times (and training requirements) are a changing. FAA Aviation News, 1-4. 
National Transportation Safety Board (NTSB). (1990). NTSB Final Report, Piper PA-46-310P, N9114B, Bristol, Indiana, May 3, 1989 (Aircraft Accident Report NTSB/CHI89FA101). Washington, DC: National Transportation Safety Board.

United States General Accounting Office. (2000). Aviation safety: Safer skies initiative has taken initial steps to reduce accident rates by 2007 (GAO/RCED-00-111). Washington, D.C. 